\title{
Nefropatia por C1q como diagnóstico diferencial de nefrite lúpica: um relato de caso
}

\author{
C1q nephropathy as a differential diagnosis of lupus nephritis: a case report
}

Nefropatía C1q como diagnosis diferenciada de la nefritis del lupus: un informe del caso

Junior Apaestegui Coriat ${ }^{1 *}$, Rafaela da Silva Almeida ${ }^{1}$, Anne Caroline da Silva Menezes ${ }^{1}$, Tayana Evelling Uchôa da Silva ${ }^{1}$, Bárbara Seabra Carneiro.

\section{RESUMO}

Objetivo: Relatar um caso de nefropatia por $\mathrm{C} 1 \mathrm{q}$ associado à infecção viral e refratário à terapia imunossupressora, além de descrever as características desta doença que a diferenciam da nefrite lúpica. Detalhamento do caso: Paciente jovem com síndrome nefrótica e diagnóstico de nefropatia por C1q estabelecido por biópsia renal, sem critérios de classificação para lúpus eritematoso sistêmico, sem resposta adequada à ciclofosfamida e micofenolato, apresentando diagnóstico de infecção por citomegalovírus ao longo da evolução, também sem melhora do quadro renal após terapia antiviral, tendo evoluído com COVID19 e desfecho desfavorável. Considerações finais: A nefropatia por C1q é uma relativamente nova entidade histopatológica das glomerulonefrites, com relatos e séries de casos mais frequentemente descritos. Apresenta manifestações clínicas diversas, sendo o diagnóstico definido pelos achados de imunofluorescência e microscopia eletrônica da biópsia renal. Corticoides são a base do tratamento, porém muitos pacientes apresentam resistência ou dependência à corticoterapia. O prognóstico é variável e depende da apresentação clínico-patológica.

Palavras-chave: Nefropatia, C1q, Lúpus.

\begin{abstract}
Objective: To report a case of $\mathrm{C} 1 \mathrm{q}$ nephropathy associated with viral infection and refractory to immunosuppressive therapy, in addition to describing the characteristics of this disease that differentiate it from lupus nephritis. Case details: Young patient with nephrotic syndrome and diagnosis of C1q nephropathy established by renal biopsy, without classification criteria for systemic lupus erythematosus, without adequate response to cyclophosphamide and mycophenolate, presenting diagnosis of cytomegalovirus infection throughout evolution, also without improvement of renal condition after antiviral therapy, having evolved with COVID19 and unfavorable outcome. Final considerations: C1q nephropathy is a relatively new histopathological entity of glomerulonephritis, with reports and series of cases more frequently described. It presents several clinical manifestations, and the diagnosis is defined by the findings of immunofluorescence and electron microscopy of renal biopsy. Corticosteroids are the basis of treatment, but many patients have resistance or dependence to corticosteroid therapy. Prognosis is variable and depends on clinical-pathological presentation.
\end{abstract}

Keywords: Nephropathy, C1q, Lupus.

\section{RESUMEN}

Objetivo: Relatar un caso de nefropatía C1q asociada a infección viral y refractaria a la terapia inmunosupresora, además de describir las características de esta enfermedad que la diferencian de la nefritis lúpica. Detalles de caso: Paciente joven con síndrome nefrótico y diagnóstico de nefropatía C1q establecido por biopsia renal, sin criterios de clasificación para lupus eritematoso sistémico, sin respuesta adecuada a ciclofosfamida y micofenolato, presentando un diagnóstico de infección por citomegalovirus a lo largo de la evolución, también sin mejoría del estado renal tras el tratamiento antiviral, habiendo

\footnotetext{
${ }^{1}$ Hospital Universitário Getúlio Vargas, Universidade Federal do Amazonas (HUGV/UFAM), Manaus - AM.

*E-mail: jrcoriat@hotmail.com
} 
evolucionado con COVID19 y resultado desfavorable. Consideraciones finales: La nefropatía de C1q es una entidad histopatológica relativamente nueva de la glomerulonefritis, con informes y serie de casos más frecuentemente descritos. Presenta varias manifestaciones clínicas, y la diagnosis es definida por los resultados de la inmunofluorescencia y de la microscopia electrónica de la biopsia renal. Los corticosteroides son la base del tratamiento, pero muchos pacientes tienen resistencia o dependencia al tratamiento con corticosteroides. El pronóstico es variable y depende de la presentación clínico-patológica.

Palabras clave: Nefropatía, C1q, Lupus.

\section{INTRODUÇÃO}

A nefropatia por C1q foi relatada pela primeira vez por Jones e Magil em 1982, recebendo a denominação de "nefrite lúpica soronegativa". Em 1985, Jennette e Hipp analisaram 800 biópsias renais e caracterizaram, nomearam e descreveram em 15 destes pacientes biopsiados, depósitos densos mesangiais na microscopia eletrônica e presença de coloração predominante para C1q na imunofluorescência; clinicamente ela se manifestava com proteinúria resistente a corticoide, pior prognóstico e nenhuma evidência clínica e/ou sorológica para Lúpus Eritematoso Sistêmico (LES), mesmo que os achados histopatológicos glomerulares se assemelhassem aos encontradas na nefrite lúpica; sendo apresentada como uma nova entidade clínico-patológica (VEGA J, et al., 2020; ZHAO Y, et al., 2014).

A prevalência da nefropatia por $\mathrm{C} 1 q$ variou entre 0,2 a 2,5\% nas amostras de biópsia renal de diferentes estudos. Em estudos na população pediátrica a prevalência variou de 2,1 a 9,2\%. Em crianças com síndrome nefrótica persistente até $16,5 \%$ das biópsias correspondiam à nefropatia por C1q. Isso provavelmente não é atribuível à incidência variável desta nefropatia, mas sim à diferença nas indicações de biópsia renal e à variação na idade de pacientes incluídos nos estudos (KANODIA KV, et al., 2015; YANG $\mathrm{YH}$, et al., 2020).

Pode apresentar distribuição bimodal por idade, afetando mais frequentemente crianças e adultos jovens e até raramente a população geriátrica, com média de idade ao redor do mundo variando entre 17 e 20 anos de idade. Há descrição de maior frequência de nefropatia por $\mathrm{C} 1 \mathrm{q}$ em afroamericanos, porém muitos dos autores não observaram predomínio racial. A distribuição por gênero é muito semelhante, com uma ligeira preponderância masculina em alguns estudos (SINHA A, et al., 2011; TARIQ N, et al., 2019).

C1q desempenha papel em vários processos imunológicos, dentre eles a tolerância imunológica, através da remoção de células apoptóticas. Receptores C1q estão presentes em diferentes tipos celulares, como as células mesangiais renais. Existem alguns fatores que poderiam explicar os depósitos de C1q, como a sua união à porção $\mathrm{Fc}$ das imunoglobulinas presentes na superfície celular, à laminina (proteína da membrana basal), a complexos imunes aprisionados inespecificamente no mesângio como resultado do aumento de fluxo de proteínas, a receptores presentes na superfície celular ou por união a células apoptóticas ou epítopos aderidos ao glomérulo (ABU-SHAHIN N, et al., 2018; VEGA J, et al., 2020).

A patogênese ainda não está bem definida. É considerada uma glomerulonefrite mediada por imunocomplexos. Outros mecanismos aventados são: anormalidades na proteína inibidora de C1q ou coloração de anticorpo apresentando reatividade cruzada com um antígeno semelhante a C1q. No entanto, o antígeno específico envolvido na nefropatia ainda não foi estabelecido (GUPTA R, et al., 2015; MALLESHAPPA P e VANKALAKUNTI M, 2013).

A nefropatia por C1q tem sido associada a infecções virais, como Epstein Barr, hepatites B e C, Vírus da Imunodeficiência Humana (HIV) e poliomavírus BK, ou raramente a neoplasias e doenças como a artrite reumatoide não controlada, com pacientes manifestando sintomas das condições subjacentes. Em alguns relatos foi encontrada associação com a síndrome de Gitelman, síndrome de Bartter, deleção do cromossomo 13 e dermatite atópica grave; esta relação pode ser explicada por predisposição genética, porém nenhum estudo molecular havia sido realizado nestes casos (DEVASAHAYAM J, et al., 2015; MALHOTRA KP, et al., 2019; MOKHTAR GA e JALALAH SM, 2015). 
O presente artigo tem como objetivo relatar o caso de uma paciente jovem com diagnóstico de nefropatia por C1q estabelecido por biópsia renal, que não preenchia os critérios de classificação para lúpus eritematoso sistêmico, sem resposta adequada à terapia instituída, tendo apresentado diagnóstico de infecção por citomegalovírus (CMV) ao longo da evolução.

\section{DETALHAMENTO DO CASO}

Paciente do sexo feminino, 19 anos, procurou um pronto socorro local em maio de 2019, com história de edema de membros inferiores há 3 dias, evolução progressiva para anasarca, dispneia aos esforços e urina espumosa. Permaneceu internada durante 6 dias para manejo de síndrome edemigênica, cilindrúria, proteinúria subnefrótica $(1,7 \mathrm{~g}$ em $24 \mathrm{~h}-$ valor de referência $[\mathrm{VR}]:<150 \mathrm{mg} / 24 \mathrm{~h})$, hipoalbuminemia $(1,4 \mathrm{~g} / \mathrm{dL}$ - VR: 3,5 a $5,2 \mathrm{~g} / \mathrm{dL}$ ) e dislipidemia (colesterol total $338 \mathrm{mg} / \mathrm{dL}-\mathrm{VR}:<200 \mathrm{mg} / \mathrm{dL}$, triglicerídeos $226 \mathrm{mg} / \mathrm{dL}$ - VR: < $150 \mathrm{mg} / \mathrm{dL}$ ); sem alteração de função renal (ureia $16 \mathrm{mg} / \mathrm{dL}$ - VR: 13-43 mg/dL, creatinina 0,68 $\mathrm{mg} / \mathrm{dL}$ - VR: 0,5-1,1 mg/dL) e sem hipertensão arterial. Hemograma apresentava-se normal e sorologias virais encontravam-se negativas. Ultrassonografia de rins e vias urinárias demonstrava ecogenicidade renal aumentada, sem alteração na diferenciação corticomedular, sugestiva de nefropatia aguda.

No período de junho a outubro de 2019 foi acompanhada pelo serviço de nefrologia do hospital universitário, que iniciou a investigação da etiologia do quadro de síndrome nefrótica (aumento na proteinúria de $24 \mathrm{~h}$ para $8,8 \mathrm{~g}-\mathrm{VR}:<150 \mathrm{mg} / 24 \mathrm{~h}$ ), com necessidade de nova internação para estabilização do quadro e diureticoterapia endovenosa. Negou hematúria, poliúria, artralgia, artrite, fotossensibilidade, lesões mucocutâneas, febre, picos hipertensivos, uso de medicamentos prévios, infecções ou internações precedentes. Previamente hígida, sem histórico familiar de nefropatias, neoplasias ou doenças autoimunes. Optou-se pela realização de biópsia renal, pulsoterapia com metilprednisolona e manutenção com prednisona $1 \mathrm{mg} / \mathrm{kg} / \mathrm{dia}$, além de início de antiproteinúrico (losartana $100 \mathrm{mg} / \mathrm{dia}$ ).

Compareceu ao serviço de reumatologia em novembro para auxílio no esclarecimento diagnóstico, com manutenção das queixas de edema de membros inferiores e dispneia, com alguma melhora após pulsoterapia, tendo surgido diminuição da diurese. Demonstrado, em pesquisa de padrão de autoimunidade, FAN (fator antinuclear) em células hep2 não reagente e anticorpo antiDNA (ácido desoxirribonucleico) dupla hélice negativo, demais autoanticorpos (antiSM, antiRNP, anticardiolipina $\operatorname{lgM}$ e $\lg G$, anticoagulante lúpico, antiRO, antiLA, crioglobulinas, $\mathrm{P}$ e C ANCA) não reagentes, complemento dentro da faixa de normalidade e Coombs direto negativo. Radiografia de tórax demonstrava congestão pulmonar, sem outros achados. Ecocardiograma sem evidência de derrame pericárdico e outras alterações estruturais.

Resultado da biópsia renal revelou glomerulonefrite proliferativa segmentar e focal à microscopia ótica (10 glomérulos acometidos dos 30 analisados) e nefrite tubulointersticial linfomononuclear discreta. À imunofluorescência depósitos glomerulares granulares de C1q (++), C3 (+) e $\lg \mathrm{M}(+)$ e cilindros de $\lg \mathrm{A}$ e C3, consistente com glomerulopatia imunomediada. O material não foi avaliado por microscopia eletrônica por indisponibilidade do método. Os achados da biópsia sugeriam nefropatia por C1q ou nefrite lúpica classe III da Organização Mundial da Saúde (OMS). Todas as medidas subsequentes de FAN hep2 e anti-DNA dupla hélice resultaram negativas. A paciente, portanto, não preenchia o critério de entrada para diagnóstico de LES, segundo os critérios de classificação EULAR/ACR, nem os critérios de classificação SLICC e ACR, exceto por proteinúria maior que $500 \mathrm{mg}$ (ARINGER M, et al., 2019; PETRI M, et al., 2012).

Devido à manutenção de síndrome nefrótica e níveis persistentemente elevados de proteinúria $(6,4 \mathrm{~g}$ em 24 horas), no período de janeiro a junho de 2020 realizou 6 doses de pulsoterapia com ciclofosfamida (CYC) e fez uso de prednisona em dose imunossupressora. Retornou em consulta ambulatorial em julho de 2020, apresentando alteração de função renal (ureia $93 \mathrm{~g} / \mathrm{dl}$ e creatinina 2,33mg/dl) e níveis persistentemente altos de proteinúria (12 e 7,6 g/24h), sendo considerada ausência de resposta ao tratamento. Decidiu-se pela realização de nova biópsia renal, cujo resultado sugeriu novamente nefropatia por C1q, com padrão à microscopia óptica de glomeruloesclerose segmentar e focal. 
Gráfico 1 - Evolução da proteinúria de 24h e creatinina sérica com o tratamento.

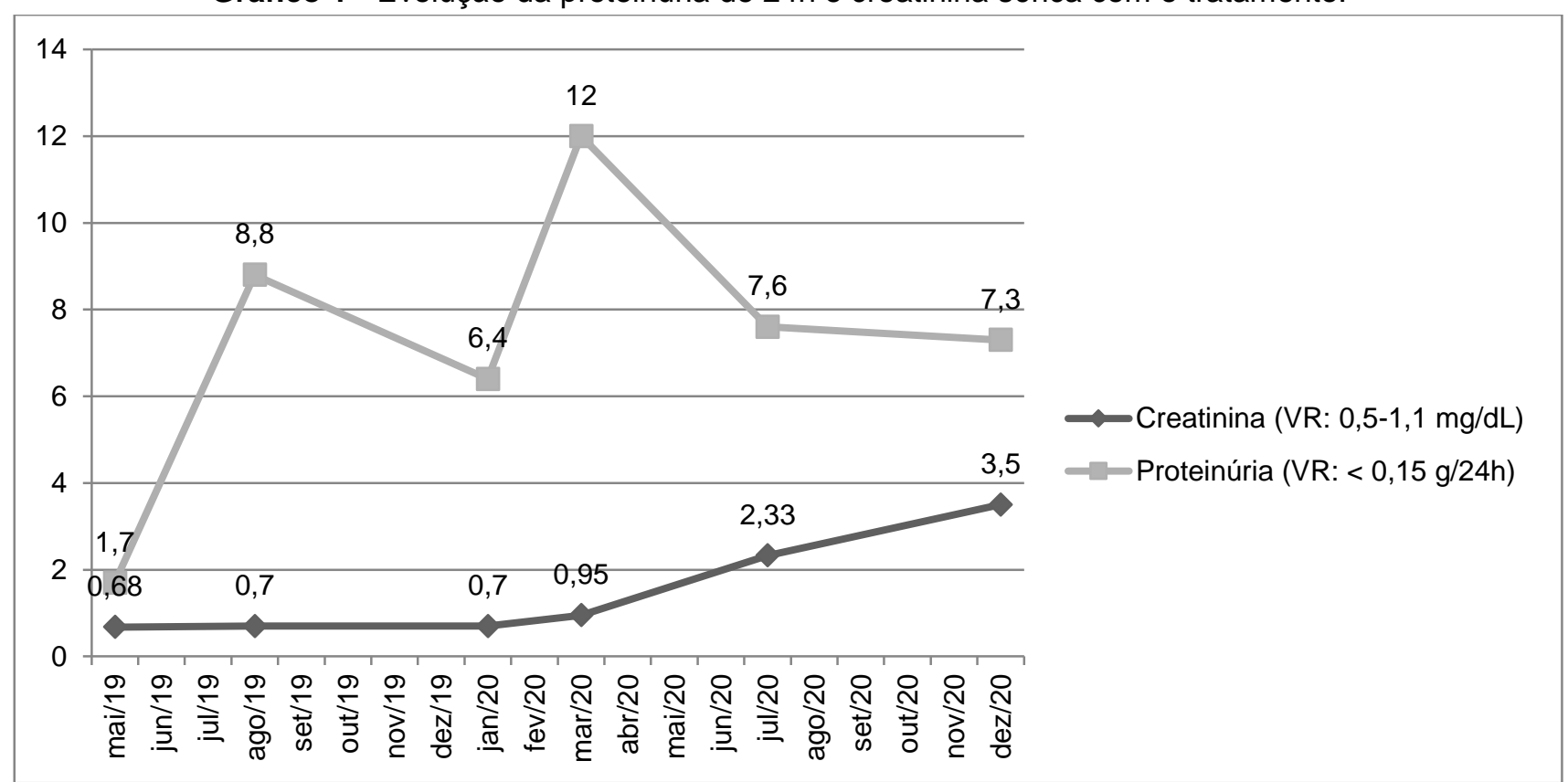

Fonte: Coriat JA, et al., 2021.

Foi identificada reativação de infecção por citomegalovírus em exames de acompanhamento (IgM positivo e em altos títulos). Quadro de citomegalovirose invasiva fora excluído através de exames de endoscopia digestiva alta, fundo de olho, tomografia de tórax e revisão da lâmina de biópsia renal. Realizado tratamento com 21 doses de valganciclovir, finalizadas em outubro de 2020. Exame de PCR qualitativo para CMV foi negativo após o término do tratamento.

Em dezembro de 2020, ainda apresentando quadro de anasarca, proteinúria em nível nefrótico (7,3 $\mathrm{g} / 24 \mathrm{~h}$ ) e diminuição da diurese associada à piora da função renal, fora prescrito micofenolato de mofetila. No entanto, em janeiro de 2021 apresentou síndrome respiratória aguda grave, tendo recebido diagnóstico de infecção por SARS-CoV2 (RT-PCR positivo), com necessidade de internação em unidade de terapia intensiva e suporte ventilatório invasivo, além de uso de droga vasoativa e terapia de substituição renal; apesar de todas as terapias impostas, evoluiu com progressiva falência multissistêmica e óbito.

\section{DISCUSSÃO}

Os critérios propostos por Jennette e Hipp em 1985 consistiam na coloração dominante ou codominante para C1q em biópsias renais de pacientes sem evidência clínica ou laboratorial de LES - FAN hep2 não reagente, anticorpos antiDNA e antiSM negativos, complemento sérico normal e sem histórico de alopecia, rash malar ou discoide, fotossensibilidade, úlceras orais, artrite, serosite, manifestações neurológicas ou transtornos psiquiátricos, como na paciente relatada. Outras glomerulopatias também devem ser excluídas, como a glomerulonefrite membranoproliferativa tipo I e a nefropatia por IgA. As colorações para C1q são frequentemente acompanhadas por IgG e $\operatorname{lgM}$, que podem ser menos ou igualmente intensas, predominantemente com depósitos densos mesangiais à microscopia eletrônica (ABU-SHAHIN N, et al., 2018; DEVASAHAYAM J, et al., 2015; MOKHTAR GA e JALALAH SM, 2015).

As características clínicas desta entidade são muito diversas e a história natural variada. As manifestações mais comumente encontradas são proteinúria na faixa não nefrótica ou síndrome nefrótica estabelecida, está associada a piores desfechos. Síndrome nefrítica, hematúria isolada e hipertensão arterial sistêmica também podem ser encontradas. A insuficiência renal aguda e a glomerulonefrite rapidamente progressiva (GNRP) são menos comuns. A heterogeneidade na apresentação pode dificultar a suspeita clínica e, portanto, o diagnóstico depende principalmente dos achados histopatológicos (ITO Y, et al., 2016; KIM K, et al., 2019; MALLESHAPPA P e VANKALAKUNTI M, 2013). 
A morfologia tem grande importância nas glomerulopatias. A nefropatia por C1q pode ser dividida em dois grupos principais com base nos padrões histopatológicos encontrados à microscopia óptica: Doença por Lesão Mínima (DLM)/Glomeruloesclerose Segmentar e Focal (GESF) e Glomerulonefrite Proliferativa (GNP). O grupo DLM/GESF, este último encontrado na paciente do caso, são podocitopatias que demonstram apagamento do processo podocitário. O grupo GNP é reconhecido como nefropatia mediada por imunocomplexos. Uma análise comparativa foi feita entre os dois grupos, não sendo encontrada diferença estatística em relação à correlação entre os padrões morfológicos e apresentações clínicas ou achados de imunofluorescência (FOGO AB, et al., 2015; JAKOVLER IR, et al., 2019; TARIQ N, et al., 2019).

A coloração para $\mathrm{C} 1 \mathrm{q}$ é evidente em todos os casos de nefropatia por $\mathrm{C} 1 \mathrm{q}$, principalmente no mesângio, com distribuição granular focal ou difusa e intensidade $\geq 2+$ (em uma escala de 0 a $4+$ ), mas também em associação com depósitos subepiteliais e/ou subendoteliais. Há possibilidade de falsa positividade da coloração para C1q em glomérulos esclerosados de forma segmentar ou global. A deposição de $\operatorname{lgG}$ e $\operatorname{lgM}$ pode ser encontrada associada em muitos casos, uma vez que fornecem ligantes para C1q na formação do complexo imune. Foi observado que pacientes com a presença de coloração difusa e extensa para C1q têm um curso clínico mais complicado, porém outras características clínicas e patológicas parecem mais importantes na definição do prognóstico (ABU-SHAHIN N, et al., 2018; DEVASAHAYAM J, et al., 2015; GUNASEKARA VM, et al., 2014).

Um padrão full house com depósitos de $\lg G$, $\lg M$, IgA, C1q e C3 foi encontrado em algumas biópsias, especialmente naquelas com morfologia de glomerulonefrite proliferativa. Nesses casos, é importante excluir a possibilidade remota de LES antes de rotular como um caso de nefropatia por C1q, porém nesta falta uma característica histopatológica geralmente presente na nefrite lúpica, que são as inclusões tubulorreticulares endoteliais. Em pacientes submetidos à nova biópsia durante sua evolução, observa-se tanto aumento dos depósitos de C1q apesar da terapia imunossupressora, como desaparecimento dos depósitos e persistência das alterações podocitárias (GUERRERO GA, et al., 2020; TARIQ N, et al., 2019; VEGA J, et al., 2020).

A nefropatia por C1q representa uma dificuldade terapêutica devido à fisiopatologia pouco compreendida e variada apresentação clínica. Não há ensaios clínicos randomizados que avaliaram o tratamento desta condição, portanto não há terapia ideal conhecida para esta doença no momento, bem como um protocolo padronizado. Contudo, a primeira linha de escolha são os corticoesteroides de forma isolada. Os pacientes que se apresentam com GESF geralmente possuem resposta insatisfatória à terapia com corticoide oral ou endovenoso e apresentam risco de desfecho desfavorável, como falência renal progressiva. No entanto, os pacientes com doença por lesão mínima e variante GN proliferativa podem responder melhor à corticoterapia, apresentando diminuição da frequência de recidivas e até mesmo remissão (MA R, et al., 2021; MALHOTRA KP, et al., 2019; RAMACHANDRAM R, et al, 2017; YANG YH, et al., 2020).

Nos casos de pacientes dependentes ou resistentes a corticoide, a pulsoterapia com metilprednisolona tem se mostrado efetiva. Além disso, um medicamento de segunda linha pode ser tentado separadamente ou em combinação com corticoide para obter remissão da doença, sendo ciclofosfamida ou ciclosporina frequentemente a segunda droga de escolha; azatioprina, micofenolato ou tacrolimus são utilizados nos casos de falha terapêutica; porém muitos pacientes apresentam recidivas frequentes apesar do uso de outros imunossupressores. Rituximabe tem apresentado resultados promissores (DEVASAHAYAM J, et al., 2015; GUNASEKARA VM et al., 2014; KIM K, et al., 2019; VEGA J, et al., 2020).

Apesar de mais de três décadas da primeira descrição, a nefropatia por C1q permanece um desafio diagnóstico e, consequentemente, uma doença pouco frequente e subdiagnosticada. Muitos autores definem esta nefropatia como "nefrite lúpica soronegativa" ou como um subgrupo de glomeruloesclerose segmentar e focal, porém com o aumento do número de relatos e séries de casos ela gradualmente vem se estabelecendo como uma entidade independente. O uso rotineiro da coloração $\mathrm{C} 1 q$ na análise de biópsia renal e a medição de anticorpos circulantes anti-C1q podem auxiliar na melhor definição diagnóstica. Mais estudos e com maior número de casos são necessários para definir a variabilidade histológica e características clínicas da nefropatia por $\mathrm{C} 1 \mathrm{q}$, bem como o manejo adequado e prognóstico desses pacientes (ABU-SHAHIN N, et al., 2018; KANODIA KV, et al., 2015; MALHOTRA KP, et al., 2019). 
A paciente do caso apresentou-se com síndrome nefrótica resistente ao tratamento com corticoide, sem resposta também com ciclofosfamida, evoluindo com infecção por citomegalovírus como intercorrência infeciosa, a qual não se pôde concluir se estava relacionada à etiologia da nefropatia ou interferiu na evolução do quadro. Fez uso por curto período de micofenolato de mofetil e evoluiu a óbito devido infecção e complicações de COVID19, não se podendo avaliar também a resposta a este tratamento. Portanto, tratase de um caso com evolução atípica de uma doença pouco prevalente, que pode trazer mais experiência e aumentar o conhecimento sobre a nefropatia por c1q.

\section{REFERÊNCIAS}

1. ABU-SHAHIN N, et al. C1q nephropathy among children with nephrotic syndrome: Ten-year experience from a pediatric nephrology unit. The Turkish Journal of Pediatrics, 2018; 60: 14-2.

2. ARINGER M, et al. 2019 EULAR/ACR Classification Criteria for Systemic Lupus Erythematosus. Arthritis Rheumatol, 2019; 71(9): 1400-1412.

3. DEVASAHAYAM J, et al. C1q Nephropathy: The Unique Underrecognized Pathological Entity. Anal Cell Pathol, 2015; 490413.

4. FOGO AB, et al. AJKD Atlas of Renal Pathology: C1q Nephropathy. Am J Kidney Dis, 2015; 66(3): e13-e14.

5. GUERRERO GA, et al. Nefropatia full house no lúpica em pediatri: reporte de dos casos. Biomédica, 2020; 40: 2207.

6. GUNASEKARA VM, et al. C1q nephropathy in children: clinical characteristics and outcome. Pediatr Nephrol, 2014; 29: 407-413.

7. GUPTA R, et al. C1q Nephropathy and Isolated CD59 Deficiency Manifesting as Necrotizing Crescentic Glomerulonephritis: A Rare Association of Two Diseases. Saudi J Kidney Dis Transpl, 2015; 26(6): 1274-1278.

8. ITO Y, et al. Successful Treatment of C1q Nephropathy by Low-Density Lipoprotein Apheresis. Ther Apher Dial, 2016.

9. JAKOVLER IR, et al. A minimum changes disease compatible with C1q nephropathy in a paediatric patient. Evolution and treatment of a difficult pathology. Nefrologia, 2019; 39(1): 84-109.

10. KANODIA KV, et al. C1q nephropathy in India: a single-center study. Saudi J Kidney Dis Transpl, 2015; 26(2): 398403.

11. KIM K, et al. C1q nephropathy in adults is a form of focal segmental glomerulosclerosis in terms of clinical characteristics. PLoS One, 2019; 14(4): e0215217.

12. MA R, et al. Case Report: Complete Remission of C1q Nephropathy Treated With a Single Low-Dose Rituximab, a Reality or Coincidence? Front. Pediatr, 2021; 8: 568773.

13. MALHOTRA KP, et al. C1q Nephropathy devoloping in a case os gastric carcinoma. Saudi J Kidney Dis Transpl, 2019; 30(6):1457-1460.

14. MALLESHAPPA P, VANKALAKUNTI M. Diverse clinical and histology presentation in c1q nephropathy. Nephrourol Mon, 2013; 5(3): 787-91.

15. MOKHTAR GA, JALALAH SM. A clinicopathological study of C1q nephropathy at King Abdulaziz University. Iran J Kidney Dis, 2015; 9(4): 279-85.

16. PETRI M, et al. Derivation and validation of the systemic lupus international collaborating clinics classification criteria for systemic lupus erythematosus. Arthritis Rheum, 2012; 64(8): 2677-2686.

17. RAMACHANDRAM $R$, et al. Successful treatment of $c 1 q$ nephropathy with CD19 targeted rituximab therapy. Nephrology, 2017; 22: 265-267.

18. SINHA a, et al. Resolution of clinical and pathologic features of C1q nephropathy after rituximab therapy. Clin Exp Nephrol, 2011; 15:164-170.

19. TARIQ N, et al. C1Q Nephropathy: A Multifaceted Disease With Infrequent Diagnosis. J Ayub Med Coll Abbottabad, 2019; $31(3):$ 308-313.

20. VEGA J, et al. Nefropatía por C1q: uma rara variedad de síndrome nefrótico. Caso Clínico. Rev Med Chile, 2020; 148: $702-706$.

21. YANG YH, et al. C1q nephropathy in a patient complicatedwith nephrotic syndrome and refractory tosteroid therapy. J Microbiol Immunol Infect, 2020; 53(1): 183-185.

22. ZHAO Y, et al. C1q nephropathy in an old woman with acute renal failure: a case report and literature review. Ren Fail, 2014; 1-3. 